\title{
Comparative study of in situ hybridization, immunohistochemistry and parasitological culture for the diagnosis of canine leishmaniosis
}

\author{
Marina C. Furtado ${ }^{1,2^{*}}$, Rodrigo C. Menezes ${ }^{1}$, Matti Kiupel ${ }^{2}$, Maria F. Madeira ${ }^{3}$, Raquel V. C. Oliveira ${ }^{4}$,
} Ingeborg M. Langohr $r^{2,5}$ and Fabiano B. Figueiredo ${ }^{1}$

\begin{abstract}
Background: The establishment of an accurate diagnostic protocol for canine visceral leishmaniosis (CanL) is a significant laboratory challenge and the lack of a reliable reference standard is one of the major problems. The aim of this study was to compare in situ hybridization (ISH), immunohistochemistry (IHC) and parasitological culture (PC) for detection of L. infantum in skin, spleen, lymph node and bone marrow of clinically healthy and sick seropositive dogs.

Findings: The study included 65 dogs positive with both DPP® and ELISA for anti-Leishmania antibodies. In situ hybridization of spleen or lymph node had the highest positivity rates of L. infantum detection. The total positivity rates for IHC, ISH and PC were $70 \%, 68.1 \%$ and $65.8 \%$, respectively. When combining techniques, the positivity rates were $81.5 \%$ in the spleen, $79.0 \%$ in lymph nodes, $59.0 \%$ in bone marrow and $52.3 \%$ in the skin. The highest percentage of infected dogs (87.7\%) was detected by using lymph node samples. When examining only skin, positivity was significantly higher in sick dogs than in the clinically healthy dogs. Infection with L. infantum was confirmed in $95.8 \%$ of sick dogs and in $82.4 \%$ of healthy dogs.
\end{abstract}

Conclusions: Considering the advantages of accurately diagnosing different Leishmania species and of being more sensitive than PC, ISH should be considered as reference standard test for the diagnosis of CanL. Spleen and lymph node are the most suitable tissues to confirm infection with L. infantum in seropositive dogs. The testing of only skin from clinically healthy dogs may result in a high percentage of false negative results.

Keywords: Leishmania infantum, Dog, Diagnosis, In situ hybridization, Immunohistochemistry, Parasitological culture

\section{Background}

Leishmanioses are caused by protozoan parasites of the Leishmania genus, that can be transmitted by phlebotomine sand flies to humans, domestic and wild mammals [1]. Leishmania infantum (syn Leishmania chagasi) is the etiological agent of zoonotic visceral leishmaniosis, for which the domestic dog represents the main reservoir in an urban environment [1].

\footnotetext{
* Correspondence: marinafurt@yahoo.com.br

'Laboratório de Pesquisa Clínica em Dermatozoonoses em Animais Domésticos, Instituto Nacional de Infectologia Evandro Chagas, Fundação Oswaldo Cruz, Rio de Janeiro, RJ, Brazil

${ }^{2}$ Department of Pathobiology and Diagnostic Investigation, College of Veterinary Medicine, Michigan State University, Lansing, MI, USA

Full list of author information is available at the end of the article
}

Enzyme-linked immunosorbent assay (ELISA), indirect immunofluorescent antibody test (IFAT) and immunochromatographic rapid test Dual Path Platform (DPP ${ }^{\circ}$ ) are used to detect anti-Leishmania antibodies in dogs [2]. Serological assays are usually applied as screening tests because of their simple execution and rapid results, although, their accuracy for detecting canine visceral leishmaniosis (CanL) is limited [2]. Parasitological culture (PC) is considered the reference standard test, detecting $L$. infantum in $62.1 \%$ to $82.2 \%$ of seropositive dogs [3, 4]. However, PC is time consuming and can be impaired by microbiological contamination [5]. Polymerase chain 
reaction (PCR) has also been used for detecting parasitic DNA in tissue samples [6]. While PCR is a valuable tool for CanL diagnosis, it does not detect viable organisms and false positive results may occur due to laboratory contamination, while false negative results can be caused by the presence of inhibitory substances [7]. In contrast, histological tests show the presence of the parasite within lesions and allow confirmation of active infection in routinely formalin fixed tissues in a safe and timely manner [7]. Immunohistochemistry (IHC) is routinely performed to detect Leishmania in tissue sections, providing more sensitivity than conventional histopathology [8-10]. In situ hybridization (ISH) using a generic [11] or a specific probe [10] has been described for the diagnosis of L. infantum infection in dogs. The specific probe had a higher sensitivity than the generic probe, IHC or conventional histopathology in skin samples [10]. Skin (SK), spleen (SP), lymph node (LN) and bone marrow (BM) are the tissues most commonly collected for detection of L. infantum in dogs, but findings regarding their sensitivity are divergent $[3,4,12-16]$.

The definition of an accurate diagnostic protocol for CanL is a significant laboratory challenge and the lack of a reliable reference standard is one of the major problems. The aim of this study was to compare the positivity rate of ISH versus IHC and PC for detection of L. infantum in SK, SP, LN and BM of clinically healthy and sick seropositive dogs.

\section{Methods}

The study population included 65 dogs identified during a serological survey performed from 2011 to 2013 in the city of Barra Mansa, state of Rio de Janeiro, Brazil. All dogs were positive in both $\mathrm{DPP}^{\circ}$ and ELISA tests for anti-Leishmania antibodies $\left(\mathrm{DPP}^{\circ} \mathrm{CVL}^{\circ}\right.$ rapid test,
BioManguinhos, Rio de Janeiro, Brazil and ELISA EIE ${ }^{\bullet}$ BioManguinhos, Rio de Janeiro, Brazil). Weight loss, alopecia, skin ulcer or nodule, exfoliative dermatitis, onychogryphosis, lymph node enlargement, splenomegaly, pale mucous membranes and skeletal muscle atrophy were considered clinical signs consistent with CanL [17]. After euthanasia, macroscopically intact SK from the scapular region, SP, popliteal LN and sternal BM were sampled for PC and the Leishmania isolates were identified by multi-locus enzyme electrophoresis [5]. For IHC and ISH, sections of SK, SP and LN and the clot of BM aspirate were fixed in $10 \%$ buffered formalin and processed as for routine paraffin embedding. The IHC was performed using an in-house rabbit polyclonal antiLeishmania serum [9]. For ISH, we used a specific antisense oligonucleotide probe as previously described [10].

Statistical analysis was performed using the Statistical Package for Social Sciences (SPSS) software for Windows (version 16.0). Infection with $L$. infantum was considered as confirmed when the parasite could be detected with at least one direct diagnostic test (PC, IHC or ISH). Fisher's exact test was used to associate positivity rates in the various tissues with the clinical status. Differences were considered significant when $p<0.05$.

\section{Ethical approval}

This study was approved by the Ethics Committee on Animal Use (LW-54/13-CEUA-FIOCRUZ).

\section{Results and discussion}

The positivity rates of Leishmania detection by IHC (Fig. 1a), ISH (Fig. 1b) and PC in SK, SP, LN and BM are listed in the Table 1. The total agreement was $88 \%$ between ISH and IHC, $77 \%$ between ISH and PC, and
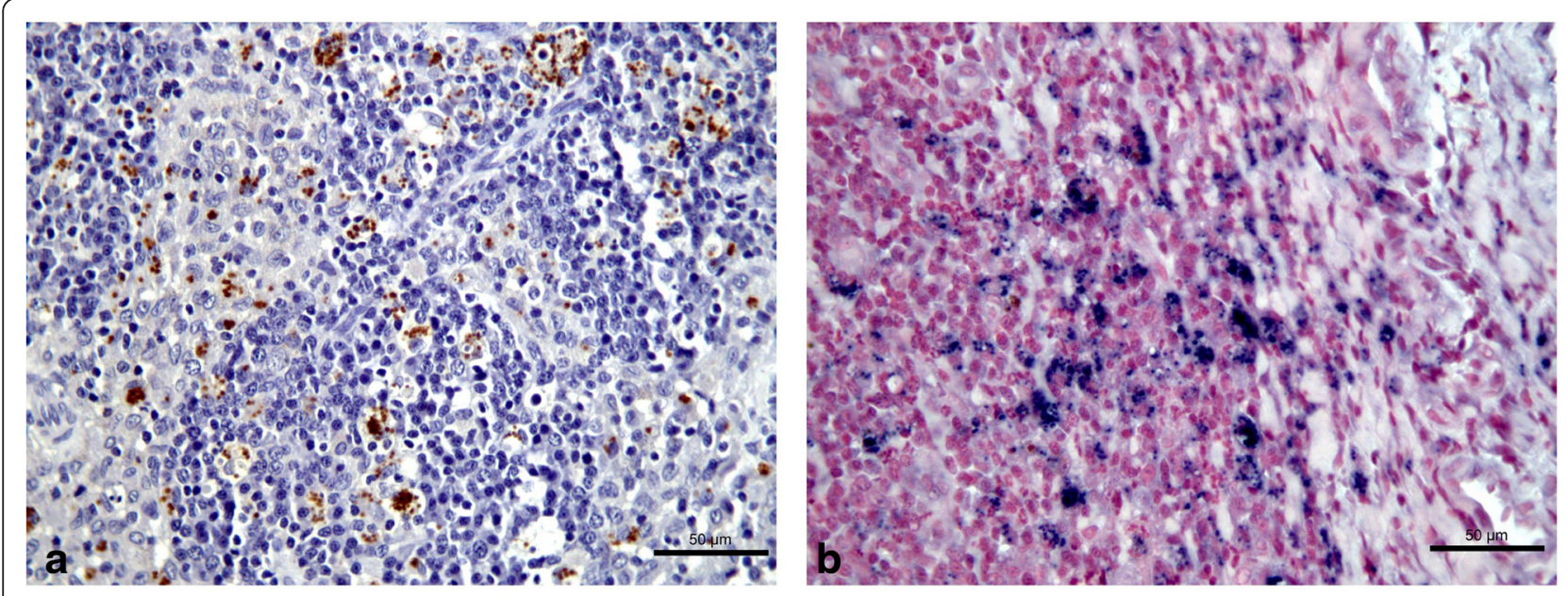

Fig. 1 Immunohistochemistry and in situ hybridization on lymph node of a dog infected with Leishmania infantum. a IHC: dark brown stained Leishmania amastigote forms within macrophages. b ISH: dark blue labeled Leishmania infantum amastigote forms within macrophages 
Table 1 Positivity rates of various diagnostic tests to confirm Leishmania infantum infection in 65 dogs

\begin{tabular}{llllll}
\hline Techniques & Skin $(n=65)$ & Spleen $(n=65)$ & Lymph node $(n=65)$ & Bone marrow $(n=65)$ & Total $(n=260)$ \\
\hline PC & $37(56.9 \%)$ & $52(80.0 \%)$ & $47(72.3 \%)$ & $35(53.8 \%)$ & $171(65.8 \%)$ \\
IHC & $34(52.3 \%)$ & $53(81.5 \%)$ & $53(81.5 \%)$ & $42(64.6 \%)$ & $182(70.0 \%)$ \\
ISH & $31(47.7 \%)$ & $54(83.1 \%)$ & $54(83.1 \%)$ & $38(58.5 \%)$ & $177(68.1 \%)$ \\
Total $(n=195)$ & $102(52.3 \%)$ & $159(81.5 \%)$ & $154(79.0 \%)$ & $115(59.0 \%)$ & $530(67.9 \%)$ \\
\hline
\end{tabular}

$P C$ parasitological culture, IHC immunohistochemistry, ISH in situ hybridization, $n$ number of examined samples

$75 \%$ between IHC and PC. The percentages of clinically healthy as well as sick seropositive dogs with active $L$. infantum infection in different tissues are presented in the Table 2.

In situ hybridization applied to SP or LN sections had the highest positivity rates of $L$. infantum detection. However, regarding the total tests performed for the four types of tissue, IHC had the highest positivity rate. Since the polyclonal antibody used for IHC is not specific for $L$. infantum species and may cross-react with fungal antigens [9], false positive results may occur. In our study, no dog was positive using only IHC testing. Furthermore, all Leishmania isolates by PC were characterized as $L$. infantum. These findings confirm that the IHC technique did not detect other Leishmania species or infectious agents that could confound our results.

One dog was negative by PC in the four tested tissues, but positive by IHC and ISH. In this case, ISH was the only technique that could identify the species of Leishmania due to the use of a specific probe. The positivity rate of Leishmania detection by PC was lower than by $\mathrm{IHC}$ or ISH. The use of PC as reference standard in CanL diagnosis is questionable because contamination or poor adaption of the parasite to the medium may impair the sensitivity of this technique and underestimate the accuracy of other tests.

Our findings suggest that SP and peripheral LN are the most suitable tissues for detection of $L$. infantum in dogs. When all test results were compared, the SP had the highest positivity rate, but LN analysis identified the highest number of infected dogs. The inferior rates of detection of L. infantum in SK and BM may be due to the lower frequency of active infection in these tissues, a possibly lower parasite load when compared to LN and SP, or the stage of infection, which was not evaluated in this study. Both $\operatorname{LN}[4,6,14,15]$ and SP $[13,16]$ have been recommended as the most suitable tissues for the diagnosis of $L$. infantum infection in dogs. Obtaining a LN biopsy is generally considered more practical and clinically safe than obtaining a SP biopsy and some professionals avoid SP sampling from live animals because of the invasiveness of the sampling technique and the risk of hemorrhage; however, a study evaluating the safety of SP aspirations in dogs, concluded that this procedure was effective and safe for the diagnosis of L. infantum infection [18].

In the present study, the testing of SK was sensitive for confirming infection in sick dogs only. Although SK is considered a suitable sample to detect $L$. infantum in dogs $[12,19]$, it should not be used in surveillance testing when the population includes clinically healthy dogs. Similarly to the sick dogs, the clinically healthy seropositive dogs had a high frequency of active $L$. infantum infection in SP, LN and BM, but a significant lower frequency in the SK. In a cross-sectional study, we cannot say whether the absence of clinical signs of CanL and SK parasitism were transitory or whether these findings are resistance characteristics. Nevertheless, as the parasite load in the SK of dogs is an indirect marker of infectiousness to the vector [20], we can assume that $53 \%$ of the clinically healthy dogs did not play a role in the transmission of $L$. infantum at the time of the sample collection.

Five dogs included in this study had negative results in all confirmatory tests performed. Although they were serologically positive in two different tests, the possibility of false positive results exists, as serological assays for the diagnosis of L. infantum infection can cross-react with other infectious agents [21, 22]. It is also reasonable

Table 2 Clinically healthy and sick seropositive dogs with confirmed Leishmania infantum infection in various tissues

\begin{tabular}{lllll}
\hline & Healthy $(n=17)$ & Sick $(n=48)$ & Total $(N=65)$ & $p$ value \\
\hline Skin & $8(47.1 \%)$ & $39(81.3 \%)$ & $47(72.3 \%)$ & 0.011 \\
Spleen & $14(82.4 \%)$ & $42(87.5 \%)$ & $56(86.2 \%)$ & 0.687 \\
Lymph node & $14(82.4 \%)$ & $43(89.6 \%)$ & $57(87.7 \%)$ & 0.421 \\
Bone marrow & $14(82.4 \%)$ & $40(83.3 \%)$ & $54(83.1 \%)$ & 1.000 \\
Confirmed infection $^{\mathrm{a}}$ & $14(82.4 \%)$ & $46(95.8 \%)$ & $60(92.3 \%)$ & 0.107 \\
\hline
\end{tabular}

${ }^{\mathrm{a}}$ Detection of Leishmania in at least one type of tissue $p<0.05$ 
to classify these dogs as "exposed", according to the criteria by Paltrinieri et al. [23], since they lived in endemic regions where $L$. infantum circulation had been confirmed. Dogs that have been exposed to L. infantum, but present no clinical signs of CanL and no evidence of active infection based on parasitological tests, may have anti-Leishmania antibodies [23].

The visceral leishmaniosis control program in Rio de Janeiro used to apply ELISA test followed by IFAT to identify dogs infected with L. infantum, but studies have shown that this protocol was inaccurate [24, 25]. Our results suggest that the protocol using $\mathrm{DPP}^{\circ}$ and ELISA has a satisfactory positive predictive value, contributing to the decrease of euthanasia of non-infected dogs due to false positive results. However, in order to properly evaluate the accuracy of this screening protocol, a multicentric and carefully designed validation study is required.

In conclusion, in situ hybridization is a valuable test for the definite diagnosis of active L. infantum infection in dogs. Considering the advantages of being able to differentiate Leishmania species in tissue samples and being more sensitive than PC, ISH should be evaluated as the future reference standard test for CanL. Spleen and LN are the most suitable tissues to confirm infection with $L$. infantum in seropositive dogs, while SK should not be used as the only sample in clinically healthy dogs.

\section{Abbreviations \\ CanL: Canine visceral leishmaniosis; PC: Parasitological culture; IHC: Immunohistochemistry; ISH: In situ hybridization; SK: Skin; SP: Spleen; LN: Lymph node; BM: Bone marrow; ELISA: Enzyme-linked immunosorbent assay; IFAT: Indirect immunofluorescent antibody test; DPP®: Dual path platform.}

\section{Competing interests}

The authors declare no competing interest.

\section{Authors' contributions}

FBF was the coordinator of this study. MCF, FBF, RCM, MK and IML designed the study. MCF carried out the physical examination of the dogs, tissue sampling and read $\mathrm{IHC}$ and ISH slides. RCM supervised the $\mathrm{IHC}$ technique. MK supervised the ISH technique. MFM carried out the parasitological culture. RVCO performed the statistical analysis. MCF drafted the manuscript. All authors read and approved the final version of the manuscript.

\section{Acknowledgements}

We thank Luiz Claudio Ferreira from INI/Fiocruz and Thomas Wood and Ricky Rosebury from DCPAH/MSU for histological technical assistance. We also thank the Secretaria Municipal de Saúde de Barra Mansa for their partnership. This study was supported by the Fundação de Amparo à Pesquisa do Estado do Rio de Janeiro (FAPERJ) (Grants: JCNE E-26/103.223/ 2011, JCNE E-26/102.247/2013 and EDNR E-26/110.598/2012). FBF and MFM hold grants from the Conselho Nacional de Desenvolvimento Científico e Tecnológico (CNPq) for productivity in research. MCF received a fellowship from FAPERJ (E-26/100.069/2013).

\section{Author details}

${ }^{1}$ Laboratório de Pesquisa Clínica em Dermatozoonoses em Animais Domésticos, Instituto Nacional de Infectologia Evandro Chagas, Fundação Oswaldo Cruz, Rio de Janeiro, RJ, Brazil. 'Department of Pathobiology and
Diagnostic Investigation, College of Veterinary Medicine, Michigan State University, Lansing, MI, USA. 'Laboratório de Vigilância em Leishmanioses, Instituto Nacional de Infectologia Evandro Chagas, Fundação Oswaldo Cruz, Rio de Janeiro, RJ, Brazil. " Laboratório de Epidemiologia Clínica, Instituto Nacional de Infectologia Evandro Chagas, Fundação Oswaldo Cruz, Rio de Janeiro, RJ, Brazil. ${ }^{5}$ Department of Pathobiological Sciences, School of Veterinary Medicine, Louisiana State University, Baton Rouge, LA, USA.

Received: 18 June 2015 Accepted: 24 November 2015

Published online: 02 December 2015

\section{References}

1. World Health Organization. Control of Leishmaniases: report of a meeting of the WHO Expert Committee on the Control of Leishmaniases, Geneva, 22-26 March 2010. WHO Technical Report Series no. 949.

2. Peixoto HM, Oliveira MRF, Romero GAS. Serological diagnosis of canine visceral leishmaniasis in Brazil: systematic review and meta-analysis. Trop Med Int Health. 2015;20(3):334-52.

3. Madeira MF, Schubach AO, Schubach TMP, Pereira SA, Figueiredo FB, Baptista C, et al. Post mortem parasitological evaluation of dogs seroreactive for Leishmania from Rio de Janeiro, Brazil. Vet Parasitol. 2006;138:366-70.

4. Mello CX, Figueiredo FB, Mendes Junior AA, Furtado MC, Miranda LFC, Madeira MF. Outbreak of canine visceral leishmaniasis in Barra Mansa, State of Rio de Janeiro. Rev Soc Bras Med Trop. 2014:47(6):788-90.

5. Almeida ABPF, Sousa VRF, Boa Sorte EC, Figueiredo FB, de Paula DAJ, Pimentel MFA, et al. Use of parasitological culture to detect Leishmania (Leishmania) chagasi in naturally infected dogs. Vector-Borne Zoonotic Dis. 2011;11(12):1555-60.

6. Almeida AB, Sousa VR, Gasparetto ND, da Silva GF, Figueiredo FB, Dutra V, et al. Canine visceral leishmaniasis: diagnostic approaches based on polymerase chain reaction employing different biological samples. Diagn Microbiol Infect Dis. 2013;76(3):321-4.

7. Maes RK, Langohr IM, Wise AG, Smedley RC, Thaiwong T, Kiupel M. Beyond H\&E: integration of nucleic acid-based analyses into diagnostic pathology. Vet Pathol. 2014;51(1):238-56.

8. Bourdoiseau G, Marchal T, Magnol JP. Immunohistochemical detection of Leishmania infantum in formalin-fixed, paraffin-embedded sections of canine skin and lymph nodes. J Vet Diagn Invest. 1997;9:439-40.

9. Quintella LP, Cuzzi T, Madeira Mde F, Okamoto T, Schubach AO. Immunoperoxidase technique using an anti-Leishmania (L.) chagasi hyperimmune serum in the diagnosis of culture-confirmed American tegumentary leishmaniasis. Rev Inst Med Trop Sao Paulo. 2009;51(2):83-6.

10. Menezes RC, Fiqueiredo FB, Wise AG, Madeira MF, Oliveira RVC, Schubach TMP, et al. Sensitivity and Specificity of in situ hybridization for the diagnosis of cutaneous infection by Leishmania infantum in dogs. J Clin Microbiol. 2013;55(1):206-11.

11. Dinhopl N, Mostegl MM, Richter B, Nedorost N, Maderner A, Fragner K, et al. In situ hybridisation for the detection of Leishmania species in paraffin wax-embedded canine tissues using a digoxigenin-labelled oligonucleotide probe. Vet Rec. 2011;169(20):525.

12. Manna L, Vitale F, Reale S, Caracappa S, Pavone LM, Morte RD, et al. Comparison of different tissue sampling for PCR-based diagnosis and follow-up of canine visceral leishmaniosis. Vet Parasitol. 2004; 125(3-4):251-62.

13. Barrouin-Melo SM, Larangeira DF, Trigo J, Aguiar PH, dos-Santos WL, Pontes-de-Carvalho L. Comparison between splenic and lymph node aspirations as sampling methods for the parasitological detection of Leishmania chagasi infection in dogs. Mem Inst Oswaldo Cruz. 2004;99:195-7.

14. Moreira MA, Luvizotto MC, Garcia JF, Corbett CE, Laurenti MD. Comparison of parasitological, immunological and molecular methods for the diagnosis of leishmaniasis in dogs with different clinical signs. Vet Parasitol. 2007;145(3-4):245-52.

15. Lima LV, Carneiro LA, Campos MB, Chagas EJ, Laurenti MD, Corbett CE, et al. Canine visceral leishmaniasis due to Leishmania (L.) infantum chagasi in Amazonian Brazil: comparison of the parasite density from the skin, lymph node and visceral tissues between symptomatic and asymptomatic, seropositive dogs. Rev Inst Med Trop Sao Paulo. 2010;52(5):259-66.

16. Solcà MS, Bastos LA, Guedes CE, Bordoni M, Borja LS, Larangeira DF, et al. Evaluating the accuracy of molecular diagnostic testing for canine visceral leishmaniasis using latent class analysis. PLoS One. 2014;9(7):e103635. 
17. Foglia Manzillo V, Di Muccio T, Cappiello S, Scalone A, Paparcone R, Fiorentino $\mathrm{E}$, et al. Prospective study on the incidence and progression of clinical signs in naïve dogs naturally infected by Leishmania infantum. PLoS Negl Trop Dis. 2013;7(5):e2225.

18. Barrouin-Melo SM, Larangeira DF, de Andrade Filho FA, Trigo J, Julião FS, Franke $C R$, et al. Can spleen aspirations be safely used for the parasitological diagnosis of canine visceral leishmaniosis? A study on asymptomatic and polysymptomatic animals. Vet J. 2006;171:331-9.

19. Madeira MF, Figueiredo FB, Pinto AG, Nascimento LD, Furtado M, Mouta-Confort E, et al. Parasitological diagnosis of canine visceral leishmaniasis: is intact skin a good target? Res Vet Sci. 2009;87(2):260-2.

20. Courtenay O, Carson C, Calvo-Bado L, Garcez LM, Quinnel RJ. Heterogeneities in Leishmania infantum infection: using skin parasite burdens to identify highly infectious dogs. PLoS Negl Trop Dis. 2014;8(1):e2583.

21. Alves AS, Mouta-Confort E, Figueiredo FB, Oliveira RVC, Schubach AO, Madeira MF. Evaluation of serological cross-reactivity between canine visceral leishmaniasis and natural infection by Trypanosoma caninum. Res Vet Sci. 2012;93:1329-33.

22. Zanette MF, Lima VM, Laurenti MD, Rossi CN, Vides JP, Vieira RF, et al. Serological cross-reactivity of Trypanosoma cruzi, Ehrlichia canis, Toxoplasma gondii, Neospora caninum and Babesia canis to Leishmania infantum chagasi tests in dogs. Rev Soc Bras Med Trop. 2014;47(1):105-7.

23. Paltrinieri S, Solano-Gallego L, Fondati A, Lubas G, Gradoni L, Castagnaro M, et al. Guidelines for diagnosis and clinical classification of leishmaniasis in dogs. J Am Vet Med Assoc. 2010;236(11):1184-91.

24. Figueiredo FB, Madeira MF, Menezes RC, Pacheco RS, Pires MQ, Furtado MC, et al. Efficacy of an indirect immunofluorescence test in the diagnosis of canine leishmaniosis. Vet J. 2010;186(1):123-4.

25. Silva DA, Madeira MF, Teixeira AC, de Souza CM, Figueiredo FB. Laboratory tests performed on Leishmania seroreactive dogs euthanized by the leishmaniasis control program. Vet Parasitol. 2011;179(1-3):257-61.

\section{Submit your next manuscript to BioMed Central} and we will help you at every step:

- We accept pre-submission inquiries

- Our selector tool helps you to find the most relevant journal

- We provide round the clock customer support

- Convenient online submission

- Thorough peer review

- Inclusion in PubMed and all major indexing services

- Maximum visibility for your research 